\title{
Ionic Liquid by Hitachi Enables In-Situ Imaging of Potable Water in IV-HRTEM
}

\author{
Marija Gajdardziska-Josifovska ${ }^{1}$, Donald Robertson ${ }^{1}$ and James P. Kilcrease ${ }^{2}$ \\ 1. Department of Physics, University of Wisconsin-Milwaukee, Milwaukee, WI 53201, USA. \\ 2. Nanotechnology Systems Div., Hitachi High Technologies America, Clarksburg, MD 20871, USA.
}

In-situ and operando electron microscopy have opened the realm of liquids for exploration under high vacuum conditions and with high-energy electrons. Liquid-cell holders provide a technical approach that allows introduction of still and flowing water in intermediate voltage high-resolution transmission electron microscopes (IV-HRTEM), but they are yet to be proven for the study of potable water. Dried macro and micro drops of drinking water contain nanoparticles that are detectable by TEM and have properties that closely resemble those of nanoparticles found in dried lake water used as source [1]. It is important to image water in its liquid state to determine if these nanoparticles are present in the water we drink or if they are created during the drying process. Here we will report challenges faced in liquid-cell experiments and present benefits of a new approach that is enabled by advances in ionic liquids.

Ionic liquids (IL) are salts with high melting points that are liquid at room temperature [2]. Unlike ordinary liquids made of neutral molecules, ionic liquids are largely made of ions and short-lived ion pairs. Hitachi's HILEM IL1000 ${ }^{\mathrm{TM}}$ is an organic salt that is designed specifically for life-sciences electron microscopy where it's increased solubility, high ionic conductivity, and non-toxic characteristics allow preservation of delicate samples in their natural forms [3]. SEM and TEM imaging with ionic liquid have been previously accomplished with voltages from $1 \mathrm{keV}$ up to $120 \mathrm{keV}$. In this research we address two important questions: can HILEM IL $1000^{\mathrm{TM}}$ withstand $300 \mathrm{keV}$ electrons and can it be used as a platform for in-situ studies of liquid drinking water?

Three types of liquid samples were prepared for imaging experiments in a Hitachi H9000NAR microscope operated at 300,000V and equipped with a Gatan Orius SC200B CCD camera. The first liquid contained a straight mix of $20 \%$ IL and $80 \%$ DI water, the second was prepared by sonication of $10 \%$ IL with $90 \%$ potable water and the third was prepared by hand mixing of $10 \%$ IL with $90 \%$ potable water. A 5 to $10 \mu \mathrm{L}$ drop of each solution was pipetted onto a copper TEM grid with holey carbon support film and excess liquid removed via wiping / blotting with lens paper. Each sample was freshly prepared prior to loading in the microscope.

Figure 1a shows a low magnification bright field TEM image of 20\% HILEM and 80\% deionized water solution. Upon insertion of the liquid sample, the vacuum in the specimen stage and gun areas showed a very minor increase from $\left(4.0 \times 10^{-7}\right.$ Torr; $\left.7.0 \times 10^{-5} \mathrm{~Pa}\right)$ to $\left(4.5 \times 10^{-7}\right.$ Torr; $\left.7.3 \times 10^{-5} \mathrm{~Pa}\right)$ respectively. Liquid menisci form on many holes in the carbon and their thickness can be assessed in comparison to empty holes. For example, the two liquid films have $\sim 88 \%$ transparency in the central areas and their image noise level $(1.4 \%)$ is only slightly higher than in vacuum $(1.25 \%)$. The liquid menisci withstand the full range of electron flux values produced by the first condenser lens in TEM mode (from $\sim 600 \mathrm{e}^{-}$ $/ \mathrm{nm}^{2} \mathrm{sec}$ at spot size 1 to $\sim 80,000 \mathrm{e}^{-/ \mathrm{nm}^{2}}$ sec at spot size 5 and magnification of $\left.\times 14,900\right)$. It is possible to break a meniscus by focusing the electron beam on its thin area resulting in dark puddles at curved hole edges. Figure $1 \mathrm{~b}$ shows a 10\% HILEM ionic liquid mixed with $90 \%$ potable water, continuing to withstand high vacuum and the $300 \mathrm{keV}$ electron irradiation. This sample area displays a wider variety of menisci thickness, from $93 \%$ transparency in the top left to $54 \%$ in top right with middle 
transparencies of $89,86,79$ and $72 \%$. The concentration of nanoparticles in potable water is low and many menisci do not contain nanoparticles (e.g. Fig. 1b).

Figure 2 demonstrates that nanoparticles exist in potable water and are not an artefact of drying. Both isolated (a) and agglomerated nanoparticles (b) are present in all samples, but the proportion changes based on the liquid mixing method. These findings can have profound implications for future water nano-purification methods.

\section{References:}

[1] FD Josifovski, TG Schneider, and M Gajdardziska-Josifovska, Microscopy \& Microanalysis (2012).

[2] Kilcrease et al. Microscopy \& Microanalysis 22, Suppl 3 (2016), p. 246.

[3] Sakaue et al. Microscopy \& Microanalysis 20, Suppl 3 (2014), p. 1012.

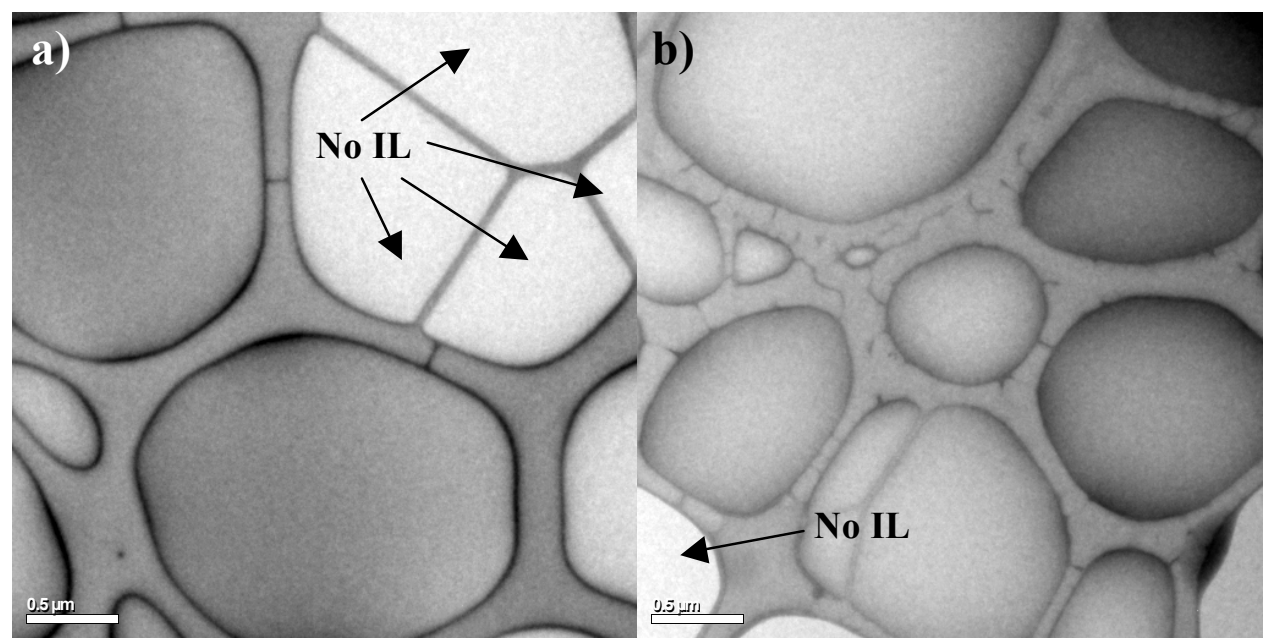

Figure 1. Bright field TEM images of liquid samples made with a) $20 \%$ HILEM IL1000 ${ }^{\mathrm{TM}}$ and $80 \%$ DI water; and b) $10 \%$ HILEM IL $1000^{\mathrm{TM}}$ and $90 \%$ potable water. Note different thicknesses of liquid menisci that fill most holes in carbon support film. Scale bar is $0.5 \mu \mathrm{m}$.

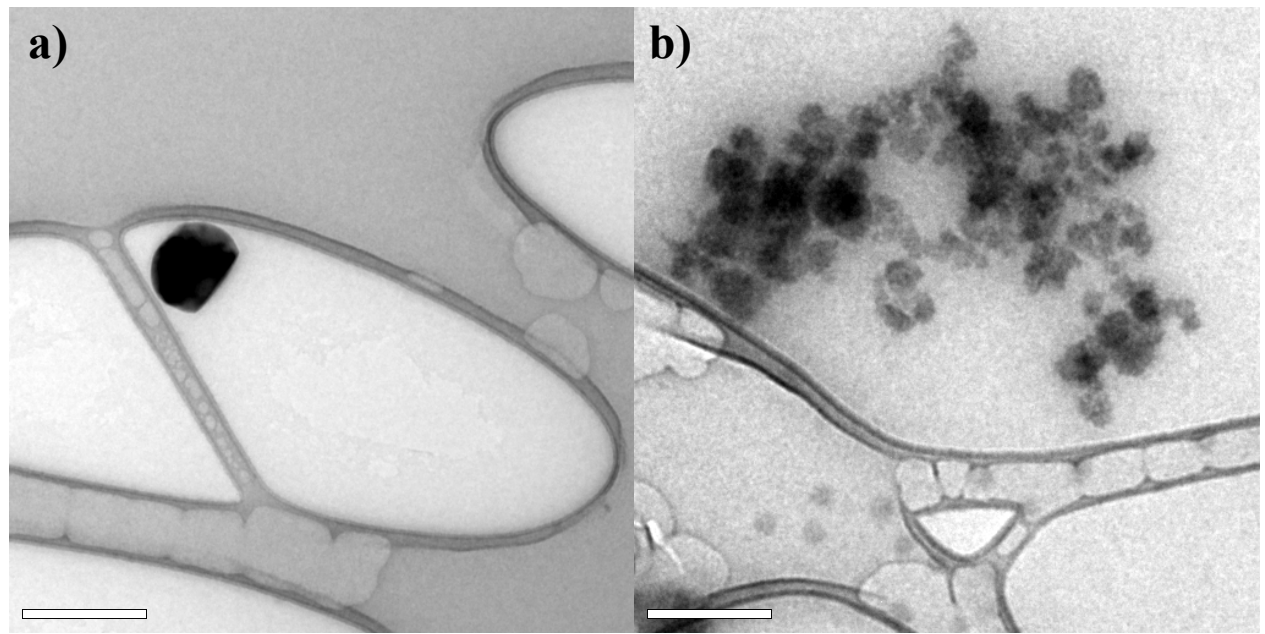

Figure 2. TEM images of naturally occurring nanoparticles suspended in liquid made with 10\% HILEM IL $1000^{\mathrm{TM}}$ and $90 \%$ potable water, mixed by: a) sonication; b) hand mixing. Scale bar is $200 \mathrm{~nm}$. 